\title{
Zum Status sprachlich kodierter Einheiten in der massenmedialen Kommunikation
}

Anna Kapuścińska (Bydgoszcz)

\begin{abstract}
This article advocates a perspective on the textuality of language units in the massmedia which is not biased with the established cultural schemes. Due to their massmedia context it appears to be unadequate to categorise them as texts, even though they are from the formal viewpoint comparable to conventional text units. However, such units are evidently not intended for the kind of reception which is typical of texts, but function rather as components of complex "visual surfaces" (Schmitz 2011: 23-42). Most researchers - also outside the field of linguistics - attempt to ascribe such units to one of the established categories of signs, so that they are perceived either as (unusual) texts or as (unusual) pictures, which inevitably results in focusing on their either textual or pictorial aspects. An alternative is an interdisciplinary approach, perceiving such units as a separate type of signs und determining their comprehensive analysis.
\end{abstract}

\section{$1 \quad$ Einleitung}

Die an Charles S. Peirce orientierte breit gefasste Semiotik, die im Folgenden mit dem Konzept von Umberto Eco illustriert wird, setzt die Auseinandersetzung dieser Disziplin mit einer enormen Varietät von Zeichen verschiedener Provenienz voraus. Daher erscheint es als berechtigt, Semiotik als ein „Etikett“ zu betrachten, ,das ein Feld von miteinander unvereinbaren Disziplinen bedeckt“ (Eco 2002: 27). Eine Folge davon ist eine umso deutlichere Binnenstrukturierung der Disziplin.

Es werden einzelne semiotische Subdisziplinen herausgegliedert, die nach der Taxonomie von Eco von den „natürlichsten“ Zeichen, wie z. B. den Zeichen in der Zoosemiotik, den Geruchssignalen oder der Kommunikation über Berührung bis hin zu den kulturellen, ästhetischen Kodes oder massenmedialen Kommunikationen (cf. Eco 2002: 20-27; cf. dazu auch Kapuścińska 2015: 67) reichen. Die wohl einzige Gemeinsamkeit zwischen diesen Zeichenkategorien und daher gleichzeitig zwischen den dafür zuständigen semiotischen Subdisziplinen besteht darin, dass sie Zeichen untersuchen (auch wenn selbst die Zeichenhaftigkeit insbesondere der „natürlichsten“ und „spontansten“ Zeichen (cf. Eco 2002: 20) mehrmals bestritten wird). Aus diesem Grund haben die einzelnen Subdisziplinen eigene und zum großen Teil souveräne Methoden herausgearbeitet, die an die jeweils spezifischen Forschungsgegenstände angepasst sind. Denn, um die Fragestellungen von Eco aufzugreifen:

Kann die Art und Weise, wie die natürlichen Sprachen untersucht werden, gleichfalls zur Erforschung von Kulturtypen und ikonischen Zeichen dienen? Gibt es einen wesentlichen Grund, so

Linguistik online 84, 5/17 - http://dx.doi.org/10.13092/lo.84.3846

CC by 3.0 
verschiedene Phänomene von verschiedenen Seiten her unter dem einen Blickwinkel der Kommunikation zu untersuchen?

(Eco 2002: 27)

Nun ist jedoch auch zu akzentuieren, dass die Kategorie von Zeichen kein statisches Gebilde ist. Im Gegenteil: Sie ist im ständigen Wandel begriffen, wodurch immer neue, von den vorhandenen Gliederungen nicht erfasste Arten von Zeichen entstehen. Insbesondere im Kontext des technologischen Fortschritts, der die Zeichenevolution intensiviert, ist dieser Tatsache in der semiotischen Reflexion zweifelsohne Rechnung zu tragen. Das grundsätzliche Ziel des vorliegenden Beitrags ist es deswegen, die Möglichkeiten der semiotischen Erforschung solcher Zeichen zu diskutieren. Dabei wird das Hauptaugenmerk auf die Zeichen in den „neuen“ Medien - hier nach Schmitz als internetgestützte Medien (cf. Schmitz 2016: 901) aufgefasst gelegt, die sprachliche Kodierung aufweisen.

\section{Zeichen-Arten im Wandel}

Von Stöckl wird zurecht auf die „heute fast allumfassend(e) Digitalisierung der Medien“ (Stöckl 2012: 21, Hervorheb. i. O.) hingewiesen. Als folgerichtige Konsequenzen der starken Durchdringung und gegenseitigen Vernetzung der technischen Medien - im technologischen Sinne (cf. Posner 1985: 256) - sieht Stöckl einerseits den Wandel der Konventionen von Textsorten und Darstellungsformen im Bemühen „Trends des jeweils fremden Mediums zu adaptieren" (Stöckl 2012: 21) und andererseits die Hybridisierung von Textsorten infolge der Medienkonvergenz.

Als ein Beispiel dafür könnten nach Fix solche Textsorten wie der Brief und die E-Mail angeführt werden, wobei es als umstritten gilt,

ob wir bei den Verlagerungen aus dem sprachlichen in ein anderes Medium von derselben Textsorte reden können, die durch das andere Medium möglicherweise spezifiziert, aber nicht in ihrem Wesen beeinträchtigt ist, oder ob wir es mit neuen Textsorten, mit neuen Bedingungen und Wirkungsmöglichkeiten zu tun haben.

(Fix 2008: 32)

Unabhängig von der Antwort auf diese Frage ist davon auszugehen, dass der Brief und die EMail sich einander mindestens in einem solchen Grad ähneln, dass sich die beiden relativ problemlos mit den linguistischen Kategorien beschreiben lassen, ohne sie zur multimodalen Perspektive ausdehnen zu müssen, was - aufgrund mehrerer methodologischer Probleme (cf. Kapuścińska 2015) - in dem vorliegenden Beitrag abgelehnt wird. Weniger selbstverständlich ist hingegen die Erfassbarkeit mit den Mitteln der (sprachlich orientierten) Linguistik solcher Einheiten, die von Stöckl in Bezug auf die Medienhybridität genannt werden, d. h. die gedruckten und Online-Zeitungen. Stöckl zufolge haben die Online-Zeitungen ,nicht nur das Design, sondern auch den sprachlichen Stil herkömmlicher Zeitungen beeinflusst“" (Stöck1 2012: 21). In Folge der Hybridisierung, die solche Ebenen wie „sprachliche Handlungen, Funktionen, stilistische Mittel, formale Gestaltung etc.“ (Stöckl 2012: 22) betreffen kann, entstehen nach Stöckl auch neue Textsorten und ,das konventionelle Spektrum der Zeitungstextsorten z. B. kommt ins Wanken“ (Stöckl 2012: 22). Dies lässt sich nur teilweise auf die Tatsache zurückführen, dass die Online-Zeitungen - die von Luginbühl eingehend diskutiert werden - auch solche Merkmale aufweisen, die in gedruckten Zeitungen nicht vorkommen können, wie „Archive und Dossiers, Links zu externen Seiten, Suchfunktionen oder interakti- 
ve Dienste wie Chat oder Diskussionsforen“ (Luginbühl/Burger 2014: 431). Von Schmitz wird unter den Unterschieden zwischen den gedruckten und Online-Zeitungen der Aspekt genannt, dass die Online-Zeitungen üblicherweise als Hypertexte gelesen werden, was einen wesentlichen Einfluss auf den Prozess ihrer Rezeption hat:

Das befreit die Hände beim Lesen, strapaziert die Augen auf andere Weise als Papierlektüre, schränkt die auf einen Blick wahrnehmbare Fläche stark ein, zieht eine mehr labyrinthische und weniger lineare oder flächige Lektüreweise nach sich und läßt eine stärkere Medienmischung (einschließlich Audio- und Videosequenzen) zu als in allen herkömmlichen Massenmedien.

(Schmitz 2001: 209)

Aus diesem Grund, ,sind die semiotischen Beziehungen zwischen den verschiedenen Zeichenarten (Text, Textdesign, Seitenlayout, Grafik, Foto, Link-Semiotik etc.) komplexer und deshalb auch schwieriger zu entschlüsseln als in Papierzeitungen." (Schmitz 2001: 209). Eine solche Tendenz, die von McLuhan als die Mosaikform der Presse diskutiert wird, soll sich „noch vor der Beschleunigung durch die Telegraphie“ (McLuhan 1992: 313) im neunzehnten Jahrhundert etabliert haben. Zweifelsohne hat sie sich aber in den heutigen ,avantgardistischen Formen der Informationsdarbietung auf Papier"“ (Schmitz 2001: 210) und noch mehr in den Online-Zeitungen radikalisiert (cf. Schmitz 2001: 210):

Dazu zählen vor allem [...] Clustering auf der Seite der inneren Textform und Flächengestaltung auf der Seite des visuellen Designs. Nicht nur in den vielfältigen weichen Randbereichen des journalistischen Info- und Entertainment, sondern längst auch in Kernbereichen der journalistischen Berichterstattung werden klassisch-lineare Textsorten durch anders geordnete Textcluster und -aggregate aufgelöst oder erweitert.

(Schmitz 2001: 210)

\section{Neue Zeichen-Art}

Es empfiehlt sich, davon auszugehen, dass auch diese Komponenten solcher Mosaiken, die aus Schriftfiguren bestehen, alles andere als herkömmliche (sprachliche) Texte sind. Vielmehr werden sie zu Elementen von Sehflächen (cf. Schmitz 2011: 23-42), indem ,[a]ngesichts steigender Informationsmengen (deswegen keineswegs wachsender Informationsqualität oder auch nur individueller Lektürezeit) [...] sich Anbieter und Leser ständig an der Grenze zu Chaos, Heterogenität, Auseinanderfall, Desorientierung und Verwirrung“ (Schmitz 2001: 210) bewegen.

Dabei handelt es sich um solche Einheiten, die - auch wenn es paradox erscheinen könnte nicht zur textüblichen Rezeption bestimmt sind. Dem Empfänger wird solche Rezeption absichtlich erschwert, indem sein Perzeptionsvermögen z. B. durch die gleichzeitige Anzeige mehrerer derartiger Einheiten (wie auf einer Zeitungs- oder Internetseite) überfordert wird (cf. Kapuścińska 2012: 117). Dadurch werden sie zu Komponenten von „Sehflächen“ reduziert und „[w]ir nehmen [sie] nicht linear wie Texte von oben links nach unten rechts, sondern nehmen das Ganze holistisch wahr, wie wir es von Bildern gewohnt sind“" (Schmitz 2011: 27). Weil die sprachlichen Texte dabei dergleichen Bearbeitung wie digitale Bilder unterzogen werden, „erlangen sie den Status von Bildern“ (Cieszkowski 2009: 312, übers. A. K.). Eine Einschränkung ist dabei, dass sie keine Distanzierung sowie kritische oder ästhetische Betrachtung zulassen (cf. Cieszkowski 2009: 312). 
Bei solcher breiten Zeichenauffassung, die hier angenommen ist, bestehen keine Zweifel, dass auch diese Einheiten als Zeichen gelten, obwohl sie manchmal als referentenlos bezeichnet werden (cf. Baudrillard 1978: 10; Cieszkowski 2014: 41). Bei solcher Bezeichnung wird zurecht davon ausgegangen, dass sie in der Regel keine materiellen Referenten haben. Infolge der Flächenschematisierung, indem jeweils neue „Texte“ in ein unveränderliches Schema inskribiert werden, wurde ,ihre repräsentative Funktion zum großen Teil beschränkt, auf das vorläufige Dasein, das Manifestieren der momentanen Anwesenheit begrenzt, die sich problemlos durch eine nächste ersetzen lässt" (Cieszkowski 2014: 41, übers. A. K.; cf. dazu auch Kapuścińska 2016: 28).

Wenn man jedoch die Perspektive von Eco einnimmt, erscheint das Fehlen eines materiellen Referenten als keine Prämisse dafür, sie aus der Zeichen-Kategorie auszugrenzen. Eco zufolge ist die Zeichenhaftigkeit nämlich von der Anwesenheit des materiellen Objekts völlig unabhängig. Die Loslösung des Zeichens von der Anwesenheit des materiellen Referenten, die das in der semiotischen Tradition fest verankerte Dreieck der Semiotik von Odgen und Richards (1923: 11) anficht, grenzt die semiotische Forschung explizit von den logischen Kategorien der Wahrheit und Lüge ab. Dadurch werden Zeichen nicht mehr an Objekte gebunden, sondern an entsprechende kulturelle Einheiten, die in einer bestimmten Kultur existieren (cf. Eco 2002: 76).

Bei einem solchen Ansatz lässt sich auch für die hierbei betrachtete Einheit ein Referent anzeigen. Dies ist nämlich der Text als kulturelle Einheit, den der Empfänger (vorausgesetzt, dass der entsprechende kulturelle Code vorhanden ist) üblicherweise erkennen kann, auch ohne den Text zu lesen. Dabei lässt sich also eine Kodierung identifizieren, die (im Gegensatz zur Kodierung ,herkömmlicher“" Texte) in einer binären Relation ihrer Anwesenheit bzw. Abwesenheit besteht.

\section{Die neue Zeichen-Art und traditionelle Methoden}

Auch wenn solche Einheiten als Zeichen von der Semiotik nicht ignoriert werden dürfen, gibt es gegenwärtig keine semiotische Subdisziplin, die dafür spezialisiert wäre. Infolgedessen lassen sich zwei mögliche Herangehensweisen in Betracht ziehen. Man könnte entweder solche neuen Hervorbringungen mit den Methoden analysieren, die sich innerhalb einer von den bereits vorhandenen Subdisziplin etabliert haben (und sie dadurch in den Forschungsgegenstand dieser Disziplin einschließen) oder potenziell eine neue Methodologie (außerhalb der tradierten semiotischen Subdisziplinen) entwickeln, die ihren Besonderheiten Rechnung tragen würde. Die beiden Lösungen sind jedoch mit einer Reihe methodologischer Probleme verbunden, die ihre effiziente Anwendbarkeit hinterfragen lassen.

In der Regel lässt sich in Bezug auf solche Zeichen eher die erste Vorgehensweise beobachten, indem sie entweder als Texte oder als Bilder betrachtet werden. Ihre Betrachtung als Texte ist auf den strukturellen Aspekt zurückzuführen. Neben den rein linguistischen Ansätzen, nach denen sie den Texten gleichzusetzen wären, sind in diesem Zusammenhang die medienwissenschaftlichen Konzepte zu erwähnen. Dabei werden die medialen Aspekte - unter Annahme des technologischen Medium-Begriffs (cf. Posner 1985: 256) - in einem deutlich größeren Ausmaß miteinbezogen, was dazu führt, dass man diese Einheiten von den herkömmlichen Texten abhebt, ohne jedoch gleichzeitig ihr Textsein zu bestreiten. Auch wenn das Kon- 
zept des „Textseins“ selbst aus der hierbei eingesetzten textlinguistischen Perspektive alles andere als eindeutig ist, gibt es Klemm zufolge ,gewisse Parameter[, die] recht häufig auftreten“. Darunter werden von Klemm folgende Parameter genannt: „Zeichenhaftigkeit, Komplexität, Strukturiertheit, Abgeschlossenheit, Ganzheit, inhaltlicher Zusammenhang, kommunikative Funktion oder Intertextualität“ (Klemm 2002: 18; cf. dazu auch Kapuścińska 2012, 2014a: 88-89). Diese definitorischen Grenzen werden in diesem Beitrag zur methodologischen Absteckung der Text-Kategorie gebraucht, obwohl sie keinesfalls als absolut betrachtet werden.

Als ein Beispiel für den Ansatz, der solche Einheiten für (auch wenn nicht herkömmliche) Texte hält, lässt sich die Konzeption von Sandbothe nennen. Darin werden drei Arten von Verflechtungen distinguiert, die im Internet zwischen den drei Komponenten der klassischen Trias (Sprache, Schrift und Bild) vorkommen. Darunter wird die „Verbildlichung der Schrift“ genannt, die (unter Berücksichtigung der textlinguistischen Terminologie) als „Verbildlichung des Textes“ bezeichnet werden könnte. Zu den „verbildlichten Texten“, die bei solcher Auffassung eine Sonderart von Texten darstellen, gehören demnach - abgesehen von den Beispielen für die „Rehabilitierung nicht-phonetischer Schriften“ (Sandbothe 1996: 425) - die Beispiele für den „bildhaften Umgang mit der phonetischen Schrift“. Dazu gehören die Hypertextdokumente, die mithilfe der HTML-Sprache so strukturiert werden, dass sie das Schreiben und Lesen zu „bildhaften Vollzügen“ machen. Der Schreibende entwickelt dabei ein „rhizomatisches Bild seiner Gedanken“ mit „einer Pluralität verschiedener Pfade und Verweisungen, die der Lesende zu neuen Gedankenbildern formt“ (Sandbothe 1996: 426). Dadurch stellt der Hypertext nach Sandbothe ein „,textuelles Bild“ oder „Textbild“ dar, in dem zusätzlich noch ,(d)ie Situierung des Textes im Raum, die taktile Auszeichnung einzelner Zeichenkomplexe als anklickbare Links, die variabel gestaltbare Struktur des Texthintergrundes oder die von Java angebotenen Möglichkeiten, Buchstaben in Bewegung zu setzen und in graphische Szenen einzubetten“ (Sandbothe 1998: 589, cf. dazu auch Kapuścińska 2014a: 84) als Beispiele für die Verbildlichung der phonetischen Schrift fungieren.

Demgegenüber lassen sich auch Beispiele dafür anführen, dass solche Einheiten als Bilder klassifiziert werden. Zwar ist auch der Bild-Begriff höchst mehrdeutig, aber, hierbei wird die (absichtlich) reduktionistische Bild-Definition von Sachs-Hombach gebraucht, die das Bild folgendermassen konzeptualisiert: „Bilder in diesem engen Sinn lassen sich als artifiziell hergestellte oder bearbeitete, flächige und relativ dauerhafte Gegenstände charakterisieren, die in der Regel innerhalb eines kommunikativen Aktes zur Veranschaulichung realer oder auch fiktiver Sachverhalte dienen“ (Sachs-Hombach 2003: 74). Zwar wird dieser Definition zurecht vorgeworfen, dass sie wichtige Bild-Arten ignoriert (cf. Scholz 2009: 115), wird davon das für diesen Beitrag relevante Bild-Konzept problemlos erfasst.

Beispielsweise betrachtet Krämer die Schriften (neben den Karten und Diagrammen bzw. Graphen) als „operative Bilder“ und widersetzt sich damit explizit der „Iconophobie“ in der französischen spätmodernen Philosophie (cf. Krämer 2009: 97), in der „,bildliche Dimension der Schrift weitgehend verhüllt und ausgeblendet blieb“ (Krämer 2009: 97). Krämer zufolge unterscheiden sich die „operativen Bilder“ (nur) darin von den herkömmlichen Bildern, dass ihr Sprachcharakter zu offensichtlich ist (cf. Krämer 2009: 95). Nach dem von Krämer verfochtenen Konzept der „Schriftbildlichkeit“ ist für die Schriftbildlichkeit der Schrifteinheiten als „materielle(n) und wahrnehmbare(n) Einschreibungen auf einer Fläche“ entscheidend, 
„dass das Bildliche zwar mit dem herkömmlichen Bild die Eigenschaft der Visualität und Zweidimensionalität teilt, abweichend von diesem aber - und hierin wiederum mit der Sprache verwandt - ein sowohl diskretes wie syntaktisches geregeltes Anordnungssystem mit referentiellen Bezügen verkörpert" (Krämer/Totzke 2012: 23).

Während die „Verschwisterung von Bild- und Sprachcharakter“ (Krämer 2009: 95), bei der „,Sprachliches' und ,Bildliches' Schriften simultan zukommt und (...) beide in keinem Verhältnis von Überordnung und Unterordnung zueinander stehen“ (Krämer/Totzke 2012: 25), als ein distinktives Merkmal der operativen Bild dargestellt wird, kann die Entscheidung, sie als Bilder (und nicht Texte) zu betrachten, als überraschend erscheinen. Sie resultiert jedoch höchstwahrscheinlich aus der „kulturhistorischen und erkenntnistheoretischen“ (Krämer 2009: 94-95) Tradition, auf die von Krämer explizit Bezug genommen wird. Demnach werden vergleichbare Phänomene ebenso als Bilder betrachtet, z. B. „,nützliche Bilder“ oder „Gebrauchsbilder“" (cf. Krämer 2009: 95).

An diesen Ansätzen ist jedoch problematisch, dass man zwangsläufig versuchen müsste, die hier thematisierten Einheiten durch das Prisma entweder der Sprachwissenschaft (darunter der Textlinguistik) oder der Bildwissenschaft zu betrachten. Obwohl die Sprachwissenschaft über geeignete Werkzeuge zur Erforschung von (sprachlichen) Texten verfügt und die (erst werdende) Bildwissenschaft die effiziente Erforschung von Bildern ermöglicht, bietet keine von den beiden Subdisziplinen der Semiotik ein methodologisches Instrumentarium, das für die ganzheitliche Erfassung dieser (,neuen“) Art von Zeichen optimal wäre.

Ohnehin wird insbesondere im Rahmen der sprachwissenschaftlichen Forschung immer häufiger versucht, das sprachwissenachaftliche Instrumentrium auch uber den Bereich der sprachlichen Texte auszudehnen. Solche Tendenzen sind vor allem in den Strömungen der sprachwissenschaftlichen Forschung erkennbar, die von dem (hierbei nicht vertretenen) breiten, semiotischen Begriff des Textes ausgehen (cf. Posner 1991: 46) ausgehen. Ein Beispiel dafür ist die „Textsemiotik“, die sich innerhalb „der Diskussion der jüngeren ostdeutschen Germanistik“ (Eckkrammer/Held 2006: 3) ansiedelt und ,für einen innovativen, grundsätzlich semiotisch verstandenen Text- und Stillbegriff" (Eckkrammer/Held 2006: 3) eintritt, sowie die damit verknüpfte „Bildlinguistik“ (cf. Eckkrammer/Held 2006: 3; cf. dazu auch Stöckl 2004).

Dennoch ist es auch bei der engeren Text-Auffassung keinesfalls eine Ausnahme, dass man in den linguistischen Herangehensweisen auch den visuellen Aspekt der geschriebenen Sprache mitberücksichtigt. Relativ selten kommen hingegen solche Ansätze vor, für die der Vorwurf zutreffend sein könnte, dass sie die Schrift „zu einem Abbild gesprochener Sprache“ (Metten 2011: 73) reduzieren. In mehreren sprachwissenschaftlichen Ansätzen wird die Medialität der Sprache deutlich aufgewertet. Selbst in den Konzepten, die den Medien-Begriff ausschließlich im technologischen Sinne auslegen, wird akzentuiert, dass das Medium nicht nur auf ein „Transportmittel“ zu reduzieren ist, weil es auch einen Einfluss auf die Botschaft ausübt (cf. Dürscheid 2011: 90).

Dabei handelt es sich jedoch jeweils um Texte, die vor allem aufgrund der sprachlichen Kodierung rezipiert werden, auch wenn das Nichtsprachliche einen unübersehbaren Beitrag zu ihrer Rezeption leistet. Das Postulat von Metten ist hingegen, eine „linguistische Ästhetik“ zu entwickeln, deren Aufgabe darin bestehen würde, ,das Ästhetische der Sprache zu beschreiben“ (Metten 2011: 75), woran nur die sichtbare Seite betrachtet wird: 
Eine Schrift, die hinsichtlich ihrer bedeutungsvollen Seite wahrgenommen wird, ist eine Zeichen-Schrift, d.h. sie wird im Kontext einer semiotischen Konzeption untersucht. Wendet man sich hingegen der sichtbaren Seite der Schrift zu, d.h. deren materieller Realisierung, so werden mediale Aspekte thematisiert.

(Metten 2011: 75)

Weil der Schrift nur bei der Wahrnehmung hinsichtlich der „,bedeutungsvollen Seite“ der Zeichen-Status zuerkannt wird, erkennt man an solcher Betrachtungsweise die - für die philologischen und semiotischen Ansätze übliche (cf. Krämer/Totzke 2012: 24) - Auffassung der Schrift als einer „Bewegung, bei der wir von der sichtbaren Textur hin zum interpretationsbasierten Gehalt des Textes gelangen, welcher dann Flucht und Zielpunkt jeden Schriftumganges zu sein scheint“ (Krämer/Totzke 2012: 24). Ob die Sprachwissenschaft dazu befähigt ist, auch die Schrifteinheiten zu analysieren, bei denen dieser „Zielpunkt“ nicht erreicht werden kann und konsequenterweise, die Schrifttypographie zum Hauptthema einer Analyse zu machen, erscheint als fraglich.

\section{Die neue Zeichen-Art und neue Methoden}

Als eine Alternative für die oben angeführten Ansätze bietet sich der Standpunkt, dass die hier thematisierten Einheiten viel zu explizite Unterschiede sowohl von den Texten als auch von den Bildern aufweisen, um mit dem für die eine oder die andere Kategorie entwickelten Instrumentarium untersucht werden zu können. Solche Annahme könnte zu einer ebenso vagen wie riskanten Konstatierung führen, dass für die Analyse solcher Einheiten eine neue semiotische Subdisziplin etabliert werden soll, die ein dafür optimales Forschungsinstrumentarium entwickeln könnte. Auch solche Sichtweise erweist sich jedoch als problematisch.

Einerseits ist zu beachten, dass sich alle wissenschaftlichen Disziplinen in der Regel über mehrere Jahrhunderte entwickeln müssen, bis sie eine Entwicklungsstufe erreichen, die man unter Umständen als hinreichend betrachten könnte, um die Einheiten, die zu ihrem Forschungsgegenstand gehören sollten, möglichst adäquat erfassen zu können. Ein geeignetes Beispiel dafür ist im Bereich der Semiotik der Werdegang von der Sprachwissenschaft. Ohne ihre Verwurzelung in einer langjährigen Tradition, die sogar bis in die Antike zurückreicht, hätte die moderne Sprachwissenschaft zweifelsohne nicht entstehen können. Der höchst mühsame und zeitaufwändige Prozess der Entstehung einer semiotischen Subdisziplin lässt sich gegenwärtig anhand von der Bildwissenschaft beobachten, wobei immer wieder diskutiert wird, ob man heutzutage überhaupt von einer etablierten Bildwissenschaft sprechen kann (cf. Sachs-Hombach 2005: 11). Eine semiotische Subdisziplin, die sich mit den hierbei besprochenen Einheiten befassten könnte, müsste theoretisch einen vergleichbaren Weg von einzelnen, zerstreuten Beobachtungen und Ansätzen durchlaufen, der zur Zeit nicht sogar eingeschlagen worden ist.

Andererseits verläuft die technologischer Entwicklung - und konsequenterweise auch solcher technologisch bedingten Einheiten - mit einer besonderen Dynamizität. Selbst für die bereits ausgereiften Disziplinen stellt der heutige technologisch bedingte Wandel ihres Untersuchungsgegenstands eine erhebliche Herausforderung dar. In der Sprachwissenschaft ist das z. B. in der Diskussion sichtbar, ob wir einen neuen Text-Begriff brauchen, die als eine Reaktion dieser Disziplin auf die „Wandelphänomene in der Welt“ (Eckkrammer 2002: 31) betrachtet wird. Es wird dabei von Eckkrammer Folgendes angemerkt:

ISSN 1615-3014 
Die Textualität des 3. Millenniums wird ohne jeden Zweifel eine essentielle Prägung durch die neuen medialen Vermittlungskanäle erfahren, die zwar primär digitale Textkonstrukte betrifft, jedoch gleichzeitig auf unseren traditionellen Textkosmos einwirken kann. Die ersten Rückkoppelungseffekte auf die klassische Schriftkultur machen sich bereits bemerkbar.

(Eckkrammer 2002: 31-32)

Ohnehin hat die Sprachwissenschaft den Vorteil, dass sie beim Auftakt der neuen, medialen Textualität bereits über ein etabliertes Instrumentarium verfügte, das mindestens für die Erfassung ihres bisherigen Forschungsgegenstandes geeignet war. Dieses konnte später problemlos als eine Basis für die Erforschung auch der neuen Phänomene gebraucht werden, denn „,[d]ie Zielsetzung bzw. Funktion der kommunikativen Akte [...] relativ unverändert [bleibt] nur die Form passt sich den Möglichkeiten der Zeit an“" (Eckkrammer 2002: 54).

Bei der Aufgabenstellung, eine Subdisziplin für die in dem Beitrag behandelte neue ZeichenKategorie (als schriftbasierte aber nicht zur textüblichen Rezeption bestimmten Einheiten) von neu auf zu erschaffen, könnte man mit diesem Vorteil nicht rechnen. Im Gegenteil, sie würde von Anfang an mit einer Fülle von Einheiten konfrontiert, die im raschen, unaufhörlichen Wandel begriffen sind. Ein dermaßen unstabiles Forschungsmaterial bietet keinen geeigneten Ausgangspunkt für die Herausbildung von Ansätzen, die genug universell wären, um nachher auch zur Erfassung neuer Einheiten gebraucht werden zu können.

\section{$6 \quad$ Traditionelle Methoden im Dienst der neuen Zeichen-Art}

Als viel empfehlenswerter erscheint aus diesen Gründen ein Ansatz, der diese Einheiten zwar nicht (allzu voreilig) als Texte oder Bilder kategorisiert, aber ihre Textualität und zugleich Bildlichkeit nicht negiert. Bei einem solchen Ansatz wird man dazu berechtigt (oder sogar ermutigt) bei der Erstellung der Methodologie aus den Leistungen der beiden bereits vorhandenen Subdisziplinen zu schöpfen, die sich nicht nur mit Texten bzw. Bildern befassen, sondern ebenso mit den Fragen der Textualität oder Bildlichkeit.

Um einen solchen transdisziplinären, aber gleichzeitig souveränen Ansatz zum Ausdruck zu bringen, können diese Einheiten mit Cieszkowski (2009: 313; 2014: 41; cf. dazu auch Kapuścińska 2014a: 86) als Text/Bilder (poln. „teksty-obrazy“, Übers. A. K.) bezeichnet werden, wobei jedoch vom Konzept der Text-Bilder als textuellen Bilder in der konkreten Poesie (cf. Gross 1994: 73) Abstand genommen wird (cf. Kapuścińska 2014a: 86, Anm. 6). Die Text/Bilder werden dabei als semiotische Einheiten definiert, die weder Texte noch Bilder sind, obwohl sie zugleich sowohl Textualität als auch Bildlichkeit aufweisen. Auf die Frage, ob in Zukunft eine „Text/Bild-Wissenschaft“" als neue semiotische Disziplin herausgearbeitet werden sollte, wird in diesem Beitrag nicht eingegangen. Wiederum ist sie keinesfalls mit der „sozial-funktionalen Text-Bild-Semiotik“ (Eder/Kühschalm 2014: 11) bzw. „Textsemiotik“ (Eckkrammer/Held 2006: 1-10) gleichzusetzen, die auf das Konzept der Multimodalität basiert sind. Bei den „Text/Bildern“ handelt sich nicht um die Wechselbeziehungen zwischen dem (sprachlichen) Text und Bild in einem multimodalen Kommunikat (cf. Adamzik 2002: 174; Dürscheid 2011: 95-97; cf. dazu Kapuścińska 2015: 66) oder multimodalen Text (cf. Stöckl 2011: 45, cf. dazu Kapuścińska 2015: 69), sondern um einzelne semiotische Einheiten, die zu keiner der tradierten semiotischen Kategorien gehören. Angesichts ihrer zunehmenden Anwesenheit in den Massenmedien ist nicht auszuschließen, dass sich in Zukunft ein solcher 
Zweig der Semiotik differenziert, auch wenn er nicht unbedingt als eine wissenschaftliche Disziplin proklamiert werden muss.

Viel wichtiger ist dennoch, dass die Text/Bilder in der semiotischen Forschung als eine eigenständige Art von Zeichen betrachtet werden, die einer speziellen, in weder Sprach- noch Bildwissenschaft vorhandenen Methodologie bedürfen. Auch wenn Beispiele für eine praktische Anwendung solcher Vorgehensweise in der einschlägigen Literatur (noch) fehlen (cf. Kapuścińska 2015: 74, Anm. 6), bietet es sich als eine höchst empfehlenswerte Richtung, eine Methodologie auf der Basis einer Zusammenstellung der in der Sprachwissenschaft und Bildwissenschaft etablierten Textualitäts- und Bildlichkeitsparameter auszubauen. Dadurch ließe sich eine Matrix erzeugen, die zu solcher Analyse der Text/Bilder gebräuchlich wäre, die ihrer sowohl Textualität als auch Bildlichkeit gleichermaßen Rechnung trägt.

\section{$7 \quad$ Fazit}

Als eine besondere Schwierigkeit aus der Perspektive der Sprachwissenschaft erscheint es bei der Betrachtung von Text/Bildern anzuerkennen, dass aus einer Buchstabenabfolge, die nach den Regeln des sprachlichen Systems gebildet wurde, das Symbolische (Sprachliche) entfernt sein kann (cf. Cieszkowski 2010: 183), wodurch sie möglicherweise kein Text (sondern ein Text/Bild) ist und daher nicht hinreichend im Rahmen der Linguistik analysiert werden kann (cf. Kapuścińska 2016: 34). Durch den unaufhörlichen Wandel der semiotischen Einheiten benötigt die Semiotik eine möglichst offene und vorurteilslose Herangehensweise an Zeichen, die zulässt, dass die betrachteten Zeichen nicht zu den tradierten Zeichen-Kategorien gehören und daher nicht im Rahmen einer tradierten semiotischen Subdisziplin (wie Sprachwissenschaft oder Bildwissenschaft) untersucht werden können, auch wenn sie z. B. strukturell gesehen den sprachlichen Zeichen ähneln.

\section{Literaturverzeichnis}

Adamzik, Kirsten (2002): „Zum Problem des Textbegriffs. Rückblick auf eine Diskussion“. In: Fix, Ulla et al. (eds.): Brauchen wir einen neuen Textbegriff? Antworten auf eine Preisfrage. Frankfurt a. M. etc., Lang: 163-182.

Baudrillard, Jean (1978): Agonie des Realen. Berlin: Merve.

Cieszkowski, Marek (2009): „O zasadzie urzeczywistnienia w języku współczesnych mediów” ('Zum Verwirklichungsprinzip in der Sprache der modernen Medien'). In: Rypel, Agnieszka/Jastrzębska-Golonka, Danuta/Sawicka, Grażyna (eds.): Język - Biznes - Media. Prace Komisji Językoznawczej Bydgoskiego Towarzystwa Naukowego tom XIX. Bydgoszcz, Bydgoskie Towarzystwo Naukowe: 309-320.

Cieszkowski, Marek (2010): „O profesjonalizmach z branży ubezpieczeniowej i symulacji jako formie komunikacji” ("Zu den Professionalismen in der Versicherungsbranche und der Simulation als Kommunikationsform'). In: Bartwicka, Halina (ed.): Język - Tekst Kultura. Prace Komisji Językoznawczej Bydgoskiego Towarzystwa Naukowego tom XX. Bydgoszcz, Bydgoskie Towarzystwo Naukowe: 179-187.

Cieszkowski, Marek (2014): „O zasadzie równoczesności w multiprzekazie” ('Zum Gleichzeitigkeitsprinzip in der Multiübertragung'). In: Sawicka, Grażyna/Czechowski, Wiesław (eds.): Sytuacja komunikacyjna i jej parametry. ,Być nadawca - być odbiorca'. Wydawnictwo, Adam Marszałek: 40-53.

ISSN 1615-3014 
Dürscheid, Christa (2011): „Medien in den Medien - Szenen im Bild. Eine pragmatische Kommunikat-Analyse“. In: Schneider, Jan Georg/Stöckl, Hartmut (eds.): Medientheorien und Multimodalität. Ein TV-Werbespot - Sieben methodische Beschreibungsansätze. Köln, von Halem: 88-108.

Eckkrammer, Eva Martha (2002): „Brauchen wir einen neuen Textbegriff?“. In: Fix, Ulla et al. (eds.): Brauchen wir einen neuen Textbegriff? Antworten auf eine Preisfrage. Frankfurt a. M. etc., Lang: 31-58.

Eckkrammer, Eva Martha/Held, Gudrun (2006): „Textsemiotik - Plädoyer für eine erweiterte Konzeption der Textlinguistik zur Erfassung der multimodalen Textrealität“". In: Eckkrammer, Eva Martha/Held, Gudrun (eds.): Textsemiotik. Frankfurt a. M. etc., Lang: 1-10. (=Sprache im Kontext 23).

Eco, Umberto (2002): Einführung in die Semiotik. Paderborn: Fink.

Eder, Franz X./Kühschelm, Oliver (2014): „Bilder - Geschichtswissenschaft - Diskurse“. In: Eder, Franz X./Kühschelm, Oliver/Linsboth, Christina (eds.): Bilder in historischen Diskursen. Wiesbaden, Springer: 3-44.

Fix, Ulla (2008): „Text und Textlinguistik“. In: Janich, Nina (ed.): Textlinguistik: 15 Einführungen. Tübingen, Narr: 15-34.

Goodman, Nelson (2012): Sprachen der Kunst. Entwurf einer Symboltheorie. Frankfurt a. M: Suhrkamp.

Gross, Sabine (1994): Lese-Zeichen. Kognition, Medium und Materialität im Leseprozeß. Darmstadt: Wissenschaftliche Buchgesellschaft.

Kapuścińska, Anna (2012): „Być albo nie być... tekstem. Problemy definicji tekstu w kontekście tekstów medialnych" ('Text-Sein oder -Nichtsein. Zu den Problemen der Textdefinition im Kontext medialer Texte'). Text und Diskurs 6/1: 121-130.

Kapuścińska, Anna (2014a): „Zur Bebilderung des Textes und Betextung des Bildes aus der text- und bildwissenschaftlichen Perspektive“. In: Antos, Gerd/Opiłowski, Roman/Jarosz, Józef (eds.): Sprache und Bild im massenmedialen Text: Formen, Funktionen und Perspektiven im deutschen und polnischen Kommunikationsraum. Wrocław/Dresden, ATUT: 8192. (= Breslauer Studien zur Medienlinguistik 1).

Kapuścińska, Anna (2014b): „Bildhaft oder bildlich? Kleiner Unterschied mit großer Wirkung“. In: Kaczmarek, Dorota et al. (eds.): Felder der Sprache. Felder der Forschung. Lodzer Germanistikbeiträge. Deutsche Sprache in linguistischen Ausprägungen. Łódź, Wydawnictwo Uniwersytetu Łódzkiego: 33-40.

Kapuścińska, Anna (2015): „Sind Texte wirklich multikodal? Zum Umfang des Text-Begriffs im Kontext massenmedialer Kommunikation“. Text und Diskurs 8/1: 63-75.

Kapuścińska, Anna (2016): „Zum semiotischen Wert der TV-Ticker“. Zeitschrift für Literaturwissenschaft und Linguistik 46/1: 25-35.

Klemm, Michael (2002): „Ausgangspunkte: Jedem seinen Textbegriff? Textdefinitionen im Vergleich“. In: Fix, Ulla et al. (eds.): Brauchen wir einen neuen Textbegriff? Antworten auf eine Preisfrage. Frankfurt a. M. etc., Lang: 17-29.

Krämer, Sybille (2009): „Operative Bildlichkeit. Von der ,Grammatologie‘ zu einer ,Diagrammatologie“? Reflexionen über erkennendes ,Sehen““. In: Heßler, Martina/Mersch, Dieter (eds.): Logik des Bildlichen. Zur Kritik der ikonischen Vernunft. Bielefeld, Transcript: 94-122. 
Krämer, Sybille/Totzke, Rainer (2012): „Einleitung. Was bedeutet ,Schriftbildlichkeit“?“. In: Krämer, Sybille/Cancik-Kirschbaum, Eva/Totzke, Rainer (eds.): Schriftbildlichkeit. Wahrnehmbarkeit, Materialität und Operativität von Notationen. Berlin, Akademie-Verlag: 1335. (= Schriftbildlichkeit 1$)$.

Luginbühl, Martin/Burger, Harald (eds.) (2014): Mediensprache. Eine Einführung in Sprache und Kommunikationsformen der Massenmedien. Berlin/Boston, de Gruyter: 425-461.

Metten, Thomas (2011): „Schrift-Bilder - Über Graffitis und andere Erscheinungsformen der Schriftbildlichkeit“. In: Diekmannshenke, Hajo/Klemm, Michael/Stöckl, Hartmut (eds.): Bildlinguistik. Theorien - Methoden - Fallbeispiele. Berlin, Schmidt: 73-93.

Ogden, Charles. K./Richards, Ivor. A. (1923/1989): The meaning of meaning, Orlando: Harvest.

Posner, Roland (1985): „Nonverbale Zeichen in öffentlicher Kommunikation: Zu Geschichte und Gebrauch der Begriffe ,verbal' und ,nonverbal', ,Interaktion' und ,Kommunikation', ,Publikum‘ und ,Öffentlichkeit', ,Medium‘, ,Massenmedium‘ und ,multimedial ““. Zeitschrift für Semiotik 7/3: 235-271.

Posner, Roland (1991): „Kultur als Zeichensystem. Zur semiotischen Explikation kulturwissenschaftlicher Grundbegriffe“. In: Assmann, Aleida/Harth, Dietrich (eds.): Kultur als Lebenswelt und Monument. Frankfurt a. M., Fischer: 37-74.

Sachs-Hombach, Klaus (2003): Das Bild als kommunikatives Medium. Elemente einer allgemeinen Bildwissenschaft. Köln: von Halem.

Sachs-Hombach, Klaus (2005): „Konzeptionelle Rahmenüberlegungen zur interdisziplinären Bildwissenschaft“. In: Sachs-Hombach, Klaus (ed.): Bildwissenschaft. Disziplinen, Themen, Methoden. Frankfurt a. M., Suhrkamp: 11-20.

Sandbothe, Mike (1996): „Bild, Sprache und Schrift im Zeitalter des Internets“. In: Hubig, Christoph/Poser, Hans (eds.): Cognitio Humana. Dynamik des Wissens und der Werte. Akten des XVII. Deutschen Kongresses für Philosophie. Workshopbeiträge Bd. I. Leipzig, Institut für Philosophie, Universität Leipzig: 421-428.

Sandbothe, Mike (1998): „Transversale Medienwelten. Philosophische Überlegungen zum Internet“. In: Vattimo, Gianni/Welsch, Wolfgang (eds.): Medien - Welten - Wirklichkeiten. München, Fink: 59-83.

Schmitz, Ulrich (2001): „Optische Labyrinthe im digitalen Journalismus“. In: Bucher, HansJürgen/Püschel, Ulrich (eds.): Die Zeitung zwischen Print und Digitalisierung. Wiesbaden, Westdeutscher Verlag: 207-232.

Schmitz, Ulrich (2011): „Sehflächenforschung. Eine Einführung“. In: Diekmannshenke, Hajo/Klemm, Michael/Stöckl, Hartmut (eds.): Bildlinguistik. Theorien - Methoden - Fallbeispiele. Berlin, Schmidt: 23-42.

Schmitz, Ulrich (2016): „Kulturwissenschaftliche Orientierung in der Medienlinguistik“. In: Jäger, Ludwig et al. (eds.): Sprache - Kultur - Kommunikation/Language - Culture - Communication: Ein internationales Handbuch zu Linguistik als Kulturwissenschaft / An International Handbook of Linguistics as a Cultural Discipline. Berlin/Boston, de Gruyter: 901-907. (= Handbücher zur Sprach- und Kommunikationswissenschaft 43).

Scholz, Oliver R. (2009): Bild, Darstellung, Zeichen. Philosophische Theorien bildlicher Darstellung. Frankfurt a. M.: Klostermann. (=Klostermann Rote Reihe 1).

Stöckl, Hartmut (2004): Die Sprache im Bild - das Bild in der Sprache. Zur Verknüpfung von Sprache und Bild im massenmedialen Text. Berlin/New York: de Gruyter. 
Stöckl, Hartmut (2011): „Sprache-Bild-Texte lesen. Bausteine zur Methodik einer Grundkompetenz“. In: Diekmannshenke, Hajo/Klemm, Michael/Stöckl, Hartmut (eds.): Bildlinguistik. Theorien - Methoden - Fallbeispiele. Berlin, Schmidt: 45-70.

Stöckl, Hartmut (2012): „Medienlinguistik. Zu Status und Methodik eines (noch) emergenten Forschungsfeldes“. In: Grösslinger, Christian/Held, Gudrun/Stöckl, Hartmut (eds.): Pressetextsorten jenseits der ,News‘. Frankfurt a. M. etc., Lang: 13-34. 